\title{
ESTUDO CLINICO-ELETRENCEFALOGRÁFICO LONGITUDINAL EM PACIENTES EPILÉPTICOS TRATADOS COM Ro 5-4023
}

\author{
MiCHEL P. LISON * \\ LAERTEL F. FASSONI**
}

\begin{abstract}
A ação do Diazepam e do Nitrazepam sôbre diversas formas clínicas de epilepsia tem sido amplamente comprovada 6, 7, 9-21, 25, 2i-30.

Estudos experimentais demonstraram significativas propriedades anticonvulsivantes de outros derivados benzodiazepínicos ${ }^{4,26}$. A baixa toxicidade dêsses compostos permite a realização de estudos terapêuticos, particularmente indicados em pacientes com manifestações epilépticas freqüentes e rebeldes às medicações habituais. No presente trabalho relatamos nossa experiência com o uso de 7-nitro-5- (2-clorofenil) -3 H-1,4-benzodiazepina 2 (1 H) -one ou Ro 5-4023.
\end{abstract}

\section{MATERIAL E METODOS}

Nossa casuistica consta de 22 pacientes com crises epilépticas freqüentes, os quais, antes do início do seguimento terapêutico, apresentavam um ou vários episódios por dia. Os dados clínicos referentes a êsses pacientes estão indicados no quadro 1 .

A inclusão de cada paciente no presente estudo só foi feita após a observação pessoal de, pelo menos, uma crise.

Os eletrencefalogramas (EEG) foram feitos em aparelho Grass, modêlo VI, re 8 canais, com eletrodos colocados segundo a posição aconselhada pela Federação Internacional para Eletrencefalografia e Neurofisiologia Clínica. A velocidade de registro do papel inscritor foi de $3 \mathrm{~cm}$ por segundo e a constante de tempo utiiizada foi de 0,05 segundo. O ganho utilizado foi de $50 \mu \mathrm{V}$ por $7 \mathrm{~mm}$ de deflexão. Em casos de potências superiores a $200 \mu \mathrm{V}$, o ganho foi reduzido.

Os traçados foram obtidos em estado de vigilia, sob ativação pela estimulação luminosa intermitente e durante o sono espontâneo ou induzido por barbitúrico (Secobarbital, $10 \mathrm{mg} / \mathrm{Kg}$ ). O EEG de cada paciente foi obtido dentro de um periodo nāo superior a dois dias antes do inicio da terapêtica com Ro 5-4023. Iniciada a medicação, os exames foram repetidos após uma e duas semanas e, a seguir, mensalmente.

Os efeitos das medicações anticonvulsivantes prèviamente administradas foram avaliados durante pelo menos um mês de observação. Verificada a sua ineficácia, associamos o benzodiazepínico. Iniciado o nôvo tratamento, a medicação

Departamento de Neurologia (Prof. J. Armbrust-Figueiredo) da Faculdade de Medicina de Ribeirão Prêto da Universidade de São Paulo: * Livre Docente;

* Residente.

Nota dos autores - Agradecemos a Produtos Roche Químicos e Farmacêuiicos S.A. pelo fornecimento do Ro 5-4023. 


\begin{tabular}{|c|c|c|c|c|c|}
\hline Caso & Sexo & Idade & Tipos de crises & $\begin{array}{l}\text { Freqüência } \\
\text { das crises }\end{array}$ & $\begin{array}{l}\text { Tratamentos } \\
\text { anteriores }\end{array}$ \\
\hline 1 & $\mathbf{M}$ & 9 a & ausências tipicas & $5-6 /$ dia & $\operatorname{sim}$ \\
\hline 2 & M & $12 \mathrm{a}$ & ausências típicas & $2-3 / \mathrm{dia}$ & $\operatorname{sim}$ \\
\hline 3 & F & $9 \mathrm{a}$ & ausências típicas & $3-5 /$ dia & $\operatorname{sim} *$ \\
\hline 4 & M & $8 \mathrm{a}$ & ausências típicas & $5-6 /$ dia & não \\
\hline 5 & $\mathrm{~F}$ & $13 \mathrm{a}$ & ausências típicas & $5-15 /$ dia & $\operatorname{sim} *$ \\
\hline 6 & $\mathrm{M}$ & $6 a$ & $\begin{array}{l}\text { ausências psicomotoras } \\
\text { com ponta-ondas lentas }\end{array}$ & $1-6 /$ dia & não \\
\hline 7 & F & 5 a & $\begin{array}{l}\text { ausências psicomotoras } \\
\text { com ponta-ondas lentas }\end{array}$ & $1-3 / d i a$ & $\operatorname{sim}$ \\
\hline 8 & $\mathbf{F}$ & $43 \mathrm{a}$ & $\begin{array}{l}\text { ausências psicomotoras } \\
\text { com foco temporal }\end{array}$ & $3-4 / \mathrm{dia}$ & $\operatorname{sim} *$ \\
\hline 9 & $\mathbf{F}$ & 13 a & ausências mioclônicas & mais de $100 /$ dia & $\operatorname{sim} *$ \\
\hline 10 & $\mathrm{M}$ & $30 \mathrm{a}$ & ausências mioclônicas & $15-70 /$ dia & $\operatorname{sim} *$ \\
\hline 11 & $\mathbf{M}$ & $14 \mathrm{a}$ & $\begin{array}{l}\text { ausências mioclônicas } \\
\text { generalizadas tônico- } \\
\text { clônicas }\end{array}$ & $\begin{array}{l}10-15 / \text { dia } \\
1 / \text { mês }\end{array}$ & $\operatorname{sim}$ \\
\hline 12 & M & $10 \mathrm{a}$ & ausências mioclônicas & $10 /$ dia & $\operatorname{sim}$ \\
\hline $\begin{array}{l}13 \\
14\end{array}$ & $\begin{array}{l}\mathrm{M} \\
\mathrm{F}\end{array}$ & $\begin{array}{l}15 a \\
39 a\end{array}$ & $\begin{array}{l}\text { ausências mioclônicas } \\
\text { mioclonias de ação } \\
\text { generalizadas tônico- } \\
\text { clônicas }\end{array}$ & $\begin{array}{l}1 \text { cada } 5 \text { a } 15 \text { minutos } \\
\text { constantes } \\
1 / \text { semana }\end{array}$ & $\operatorname{sim} *$ \\
\hline 15 & M & $7 \mathrm{a}$ & acinéticas & $4-5 /$ dia & $\operatorname{sim}$ \\
\hline 16 & M & 2 a $7 \mathrm{~m}$ & ausências atípicas & $8-10 /$ dia & $\operatorname{sim} *$ \\
\hline 17 & M & $4 a$ & acinéticas & 1 cada 10 minutos & $\operatorname{sim} *$ \\
\hline 18 & $\mathbf{M}$ & $4 \mathrm{a}$ & $\begin{array}{l}\text { ausências atípicas } \\
\text { tônicas axiais } \\
\text { mioclonias }\end{array}$ & $\begin{array}{l}3-5 / \text { dia } \\
1 / \text { dia } \\
1-2 / \text { dia }\end{array}$ & $\operatorname{sim} *$ \\
\hline 19 & $\mathbf{M}$ & $8 \mathrm{a}$ & $\begin{array}{l}\text { acinéticas } \\
\text { hemiclonias } \\
\text { tônicas axiais }\end{array}$ & $\begin{array}{l}10-15 / \text { dia } \\
4-\quad 5 / \text { semana } \\
1 / \text { semana }\end{array}$ & $\operatorname{sim}$ \\
\hline 20 & $F$ & 5 a & $\begin{array}{l}\text { acinéticas } \\
\text { tônico-clônicas gene- } \\
\text { ralizadas }\end{array}$ & $\begin{array}{l}1 \text { cada } 10 \text { minutos } \\
1 \text { cada } 5-6 \text { meses }\end{array}$ & $\operatorname{sim} *$ \\
\hline 21 & $\mathrm{~F}$ & $7 \mathrm{a}$ & $\begin{array}{l}\text { acinéticas } \\
\text { tônicas axiais }\end{array}$ & $\begin{array}{r}15-20 / \text { dia } \\
4-5 / \text { dia }\end{array}$ & $\operatorname{sim} *$ \\
\hline 22 & $\mathrm{M}$ & $4 a$ & $\begin{array}{l}\text { acinéticas } \\
\text { hemiclônicas }\end{array}$ & $\begin{array}{l}3-5 / \text { dia } \\
0-1 / \text { dia }\end{array}$ & $\operatorname{sim} *$ \\
\hline
\end{tabular}

Quadro 1 - Dados clinicos dos 22 pacientes antes do tratamento com Ro 5-4023.. * Tratamentos anteriores com outros derivados benzodiazepínicos. 
pré-existente foi mantida ou diminuida; a dosagem de barbitúricos e/ou primidone foi aumentada naqueles casos em que crises convulsivas generalizadas ocorreram durante o tratamento.

A dose de benzodiazepinico foi mantida dentro dos limites individuais de tolerância, sendo administrada cada 8 horas. Não se administrou dose maior que $6 \mathrm{mg} /$ dia, a não ser na paciente com mioclonias de ação (caso 14) e num paciente com ausências miclônicas (caso 10) que receberam, por tempo limitado, $12 \mathrm{mg} /$ dia.

A avaliaçāo do número de crises antes e durante as diversas fases do tratamento foi realizada através de um diário feito pelos familiares ou pelo próprio paciente. Insistiu-se particularmente sôbre a necessidade de vigilância contínua.

\section{RESULTA DOS}

Ação do Ro 5-4023 sôbre as manifestações epilépticas - No quadro 2 estão indicados os resultados clínicos globais.

R e s u l t a d o s

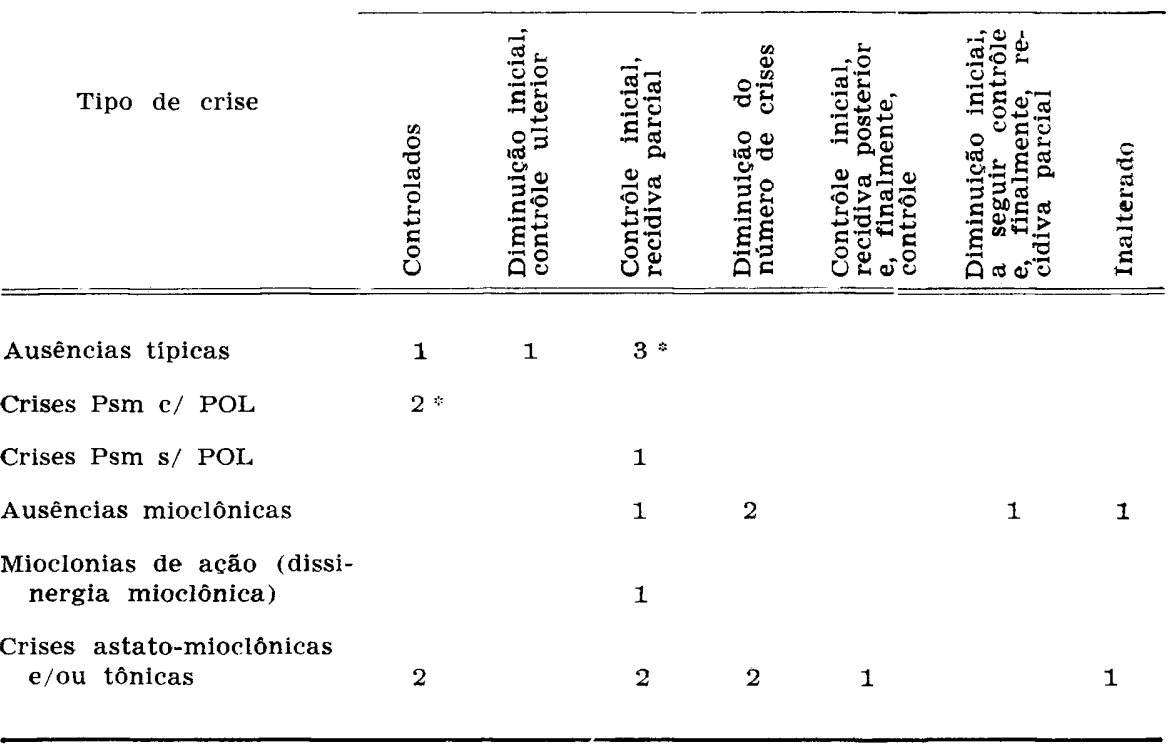

Quadro 2 - Resultados obtidos nos 22 pacierites tratados com Ro 5-4023. * Um paciente com ausências tipicas e um paciente com crises Psm com ponta-ondas lentas não foram tratados anteriormente com outros produtos. Legenda: crises Psm com $P O L=$ crises psicomotoras com ponta-ondas lentas; crises Psm $s / P O L=$ crises psicomotoras sem ponta-ondas lentas.

No quadro 3 estão indicados os efeitos da medicação benzodiazepinica sôbre a frequiência das crises durante o seguimento terapêtico nos 5 pacientes com ausências tipicas. 


\begin{tabular}{|c|c|c|c|}
\hline Caso & Freqüência das crises & $\begin{array}{l}\text { Doses em- } \\
\text { pregadas } \\
\text { (em mg) }\end{array}$ & $\begin{array}{l}\text { Duração } \\
\text { do } \\
\text { seguimento }\end{array}$ \\
\hline 1 & $\begin{array}{l}\text { Diminuição maior que } 75 \% \text { : primeira semana } \\
\text { Sem crises em seguida }\end{array}$ & $\begin{array}{l}1,5 \\
3,0\end{array}$ & 8 meses \\
\hline 2 & $\begin{array}{l}\text { Sem crises durante uma semana } \\
\text { Diminuição maior que } 75 \% \text { : uma semana } \\
\text { Diminuição menor que } 50 \% \text { : uma semana } \\
\text { Recidiva uma semana } \\
\text { Sem crises: uma semana } \\
\text { Diminuição maior que } 75 \% \text { : uma semana } \\
\text { Sem crises: } 6 \text { semanas } \\
\text { Diminuição maior que } 75 \%: 4 \text { meses }\end{array}$ & $\begin{array}{r}3,0 \\
3,0 \\
3,0 \\
- \\
4,0 \\
4,0 \\
6,0 \\
6,0\end{array}$ & 7 meses \\
\hline 3 & Sem crises desde a primeira semana & 3,0 & 10 meses \\
\hline 4 & $\begin{array}{l}\text { Sem crises: uma semana } \\
\text { Recidiva: uma semana } \\
\text { Diminuição maior que } 75 \%: 4 \text { semanas } \\
\text { Sem crises: } 6 \text { semanas } \\
\text { Diminuição maior que } 75 \%: 5 \text { meses }\end{array}$ & $\begin{array}{c}3,0 \\
- \\
3,0 \\
4,0 \\
4,0\end{array}$ & 8 meses \\
\hline $\bar{\jmath}$ & $\begin{array}{l}\text { Sem crises: } 4 \text { semanas } \\
\text { Diminuição maior que } 75 \%: 3 \text { meses } \\
\text { Diminuição de } 50 \text { a } 75 \%: 3 \text { meses }\end{array}$ & $\begin{array}{l}6,0 \\
6,0\end{array}$ & 7 meses \\
\hline
\end{tabular}

Quadro 3 - Freqüência das crises durante o seguimento terapêtico com Ro 5-4023 nos 5 pacientes com ausências tipicas. Legenda: $-=$ suspensão da medicação.

No quadro 4 estão indicados os efeitos da droga sôbre a incidência das crises durante o seguimento terapêutico nos três pacientes com crises psicomotoras.

\begin{tabular}{ccccc}
\hline Caso & Freqüência das crises & $\begin{array}{c}\text { Doses em- } \\
\text { pregadas } \\
\text { (em mg) }\end{array}$ & $\begin{array}{c}\text { Duração } \\
\text { do } \\
\text { seguimento }\end{array}$ \\
\hline \hline 6 & Sem crises desde a primeira semana & 3,0 & 8 meses \\
8 & Sem crises desde a primeira semana & 3,0 & 2 meses \\
& Sem crises: duas semanas & 6,0 & 2 meses \\
& Diminuição maior que $75 \%:$ duas semanas & 6,0 & 6,0 & \\
\hline
\end{tabular}


No quadro 5 estão indicados os efeitos da droga sôbre a incidência das crises durante o seguimento terapêutico nos 5 pacientes com ausências mioclônicas.

\begin{tabular}{|c|c|c|c|}
\hline Caso & Freqüência das crises & $\begin{array}{c}\text { Doses em- } \\
\text { pregadas } \\
\text { (em mg) }\end{array}$ & $\begin{array}{c}\text { Duração } \\
\text { do } \\
\text { seguimento }\end{array}$ \\
\hline \multirow[t]{3}{*}{9} & Diminuição maior que $75 \%$ : duas semanas & 3,0 & 7 meses \\
\hline & Sem crises: três semanas & 3,0 & \\
\hline & Diminuição maior que $\mathbf{7 5} \%$ : $\mathbf{5}$ meses & 3,0 & \\
\hline 10 & Diminuição de $50-80 \%: 10$ meses & 6,0 & 10 meses \\
\hline & $\begin{array}{l}\text { A suspensão, por dias, após } 3 \text { e } 9 \text { meses levou } \\
\text { à recidiva das crises }\end{array}$ & & \\
\hline 11 & Sem crises: uma semana & 6,0 & 6 meses \\
\hline & Diminuição maior que $75 \%: 6$ meses & a $\quad 4,0$ & \\
\hline 12 & Inalterado & 4,0 a 12,0 & 3 semanas \\
\hline 13 & Diminuição maior que $75 \%$ & 6,0 & 5 meses \\
\hline
\end{tabular}

Quadro 5 - Freqüência das crises durante o seguimento terapêtico nos 5 pacientes com ausências mioclônicas.

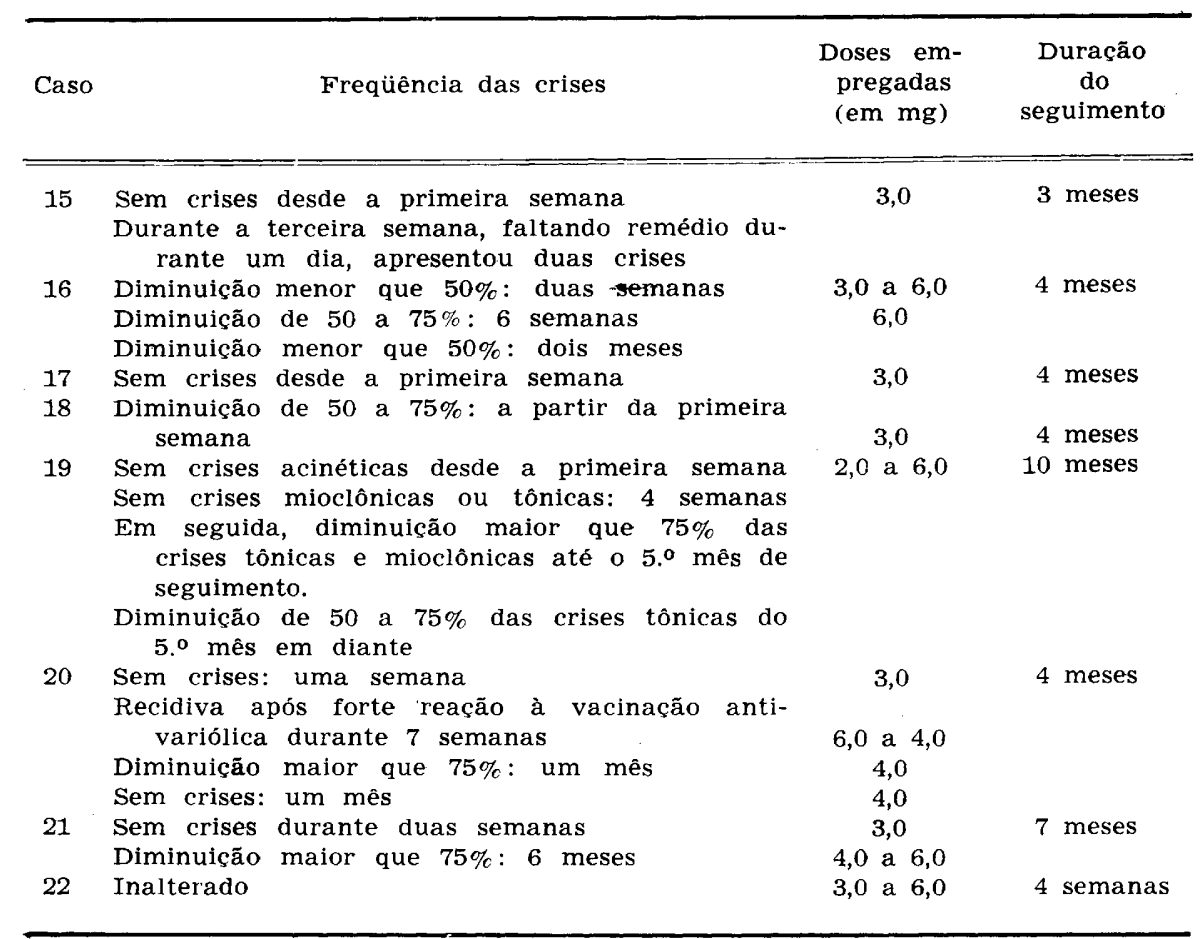

Quadro 6 - Freqüência das crises durante o seguimento terapêtico nos 8 pacientes com sindrome de Lennox. 
A paciente com mioclonias de ação (dissinergia cerebelar mioclônica, caso 14) não apresentou manifestação durante três semanas com $6 \mathrm{mg}$ de Ro 5-4023. Houve, a seguir, discretas mioclonias à movimentação voluntária, com ligeira acentuação, durante três meses. A elevação da dose para $12 \mathrm{mg} / \mathrm{dia}$ permitiu novamente o contrôle das manifestações por duas semanas, havendo recidiva parcial. Contudo, até o final do seguimento terapêutico ( 6 meses) a paciente era capaz de se alimentar, de se vestir e de se pentear, o que não conseguia fazer antes de medicada.

No quadro 6 estão indicados os efeitos da droga sôbre a incidência das manlfestações epilépticas nos 8 pacientes com síndrome de Lennox.

Efeitos colaterais - No quadro 7 estão sintetizados os principais efeitos colaterais observados em 11 dos 22 pacientes.

\begin{tabular}{|c|c|c|c|c|}
\hline Efeito colateral & $\begin{array}{l}\text { N.o de } \\
\text { casos }\end{array}$ & Intensidade & $\begin{array}{l}\text { Persis- } \\
\text { tente }\end{array}$ & $\begin{array}{l}\text { Passa- } \\
\text { geiro }\end{array}$ \\
\hline Sonolência & 7 & $+++a+t+t$ & & $\operatorname{sim}$ \\
\hline Hipotonia & 4 & $+t a+t+t$ & & $\operatorname{sim}$ \\
\hline Ataxia & 2 & $+++a+++$ & & $\operatorname{sim}$ \\
\hline $\begin{array}{l}\text { Precipítação crises convul- } \\
\text { sivas generalizadas }\end{array}$ & 2 & ++ & $\operatorname{sim}$ & \\
\hline Distúrbio de comportamentc & 1 & ++ & & $\operatorname{sim}$ \\
\hline Vômitos & 3 & +++ & & $\operatorname{sim}$ \\
\hline Confusão mental & 1 & ++ & & sim \\
\hline Tontura & 3 & $++a+t+$ & & $\operatorname{sim}$ \\
\hline
\end{tabular}

Quadro 7 - Principais efeitos colaterais observados em 11 dos 22 pacientes.

Evolução eletrencefalográfica - A evolução eletrencefalográfica dos 5 pacientes com ausências típicas está resumida no quadro 8 .

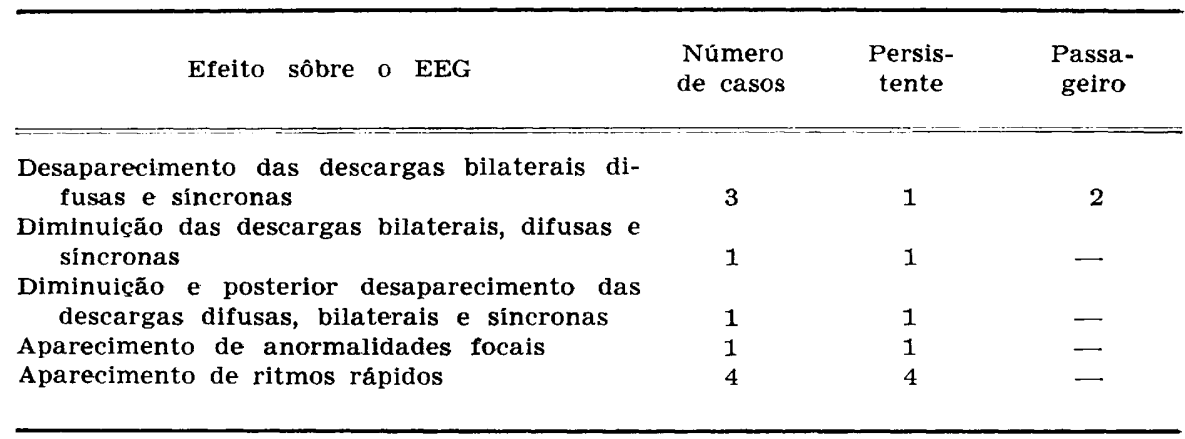

Quadro 8 - Evolução eletrencefalográfica dos 5 pacientes com ausências tipicas.

Os EEG dos dois pacientes com crises psicomotoras e com ponta-ondas lentas evidenciaram desaparecimento das descargas difusas e persistência das focais. $O$ EEG da paciente com crise psicomotora sem ponta-ondas lentas mostrou diminui- 
cão de intensidade das descargas temporais; contudo, a partir da segunda semana de tratamento, houve ligeira acentuação destas mesmas descargas.

No quadro 9 estão resumidas as principais modificações do EEG dos 5 pacientes com ausências mioclônicas.

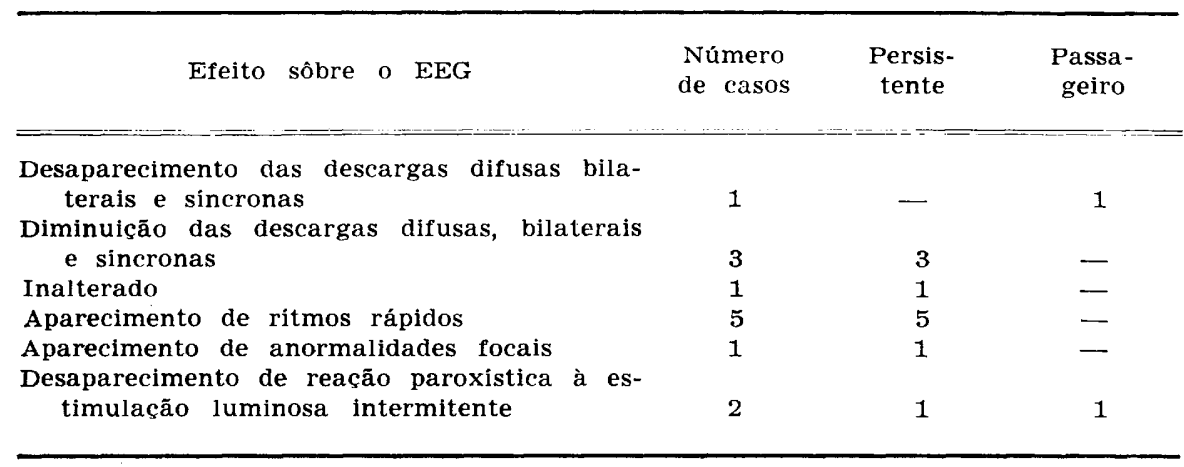

Quadro 9 - Evolução eletrencefalográfica dos 5 pacientes com ausências miclônicas.

Na paciente com mioclonias de ação (caso 14), os EEG realizados no primeiro mês de tratamento não evidenciaram descargas difusas. Raros complexos poliponta-ondas lentas surgiram em seguida. Coincidindo com o aumento da dosagem da droga, houve novamente o desaparecimento passageiro das anormalidades difusas. A reação paroxística à estimulação luminosa intermitente observada antes tes do seguimento terapêutico não foi mais observada enquanto tratada com Ro 5-4023.

No quadro 10 estão resumidas as principais modificações do EEG dos 8 pacientes com sndrome de Lennox.

\begin{tabular}{lccc}
\hline Efeito sôbre o EEG & $\begin{array}{c}\text { Número } \\
\text { de casos }\end{array}$ & $\begin{array}{c}\text { Persis- } \\
\text { tente }\end{array}$ & $\begin{array}{c}\text { Passa- } \\
\text { geiro }\end{array}$ \\
\hline \hline $\begin{array}{l}\text { Desaparecimento das descargas de ponta-on- } \\
\text { das lentas difusas }\end{array}$ & 4 & 2 & 2 \\
$\begin{array}{l}\text { Diminuicão das descargas de ponta-ondas } \\
\text { lentas }\end{array}$ & 2 & 2 & - \\
$\begin{array}{l}\text { Aparecimento de anormalidades focais } \\
\text { Aparecimento de descargas de pontas rítmi- } \\
\text { cas difusas }\end{array}$ & 2 & 2 & 1 \\
$\begin{array}{l}\text { Aparecimento de rítmos rápidos } \\
\text { Inalterado }\end{array}$ & 5 & 5 & 2 \\
\hline
\end{tabular}

Quadro 10 - Evolução eletrencefalográfica dos 8 pacientes com sínarome de Lennox.

\section{OMENTARIOS}

Nossos achados demonstram ter ocorrido rápida e significativa redução de intensidade e freqüência das diversas manifestaçōes epilépticas em 20 dos 22 casos. A análise de nossos resultados mostra que o insucesso de 
tratamentos anteriores com anticonvulsivantes usuais ou mesmo com Diazepam ou Nitrazepam, não influiram nos resultados da terapêutica com Ro 5-4023.

A ação anticonvulsivante é obtida com doses sensìvelmente inferiores àquelas comumente empregadas com o Diazepam e o Nitrazepam. É interessante assinalar que os 5 casos controlados desde o inicio do seguimento terapêutico, e cujas idades variavam entre 4 e 9 aros, foram tratados com apenas $3 \mathrm{mg} /$ dia. Os dois pacientes não beneficiados receberam doses mais elevadas. Tal observação parece indicar que aqueles casos que não respondem de modo favorável no início do tratamento, não serão influenciados mesmo que haja elevação da dosagem empregada.

Ao contrário dos outros derivados benzodiazepínicos, particularmente o Diazepam ${ }^{6}$, não há necessidade de fracionar a administração da droga em mais de três tomadas por dia.

Contudo, em dois pacientes com síndrome de Lennox, foram observadas crises tônicas durante a última fase do sono. A influência facilitadora do sono sôbre tais manifestações é conhecida ${ }^{5}$, constituindo indicação de refôrço da medicação para o periodo noturno.

Uma vez iniciado o ensaio terapêtico, a freqüência e a intensidade das crises dependeram de dois fatôres principais: a dosagem do medicamento e intercorrência de processos infecciosos.

A supressão precoce do Ro 5-4023, mesmo por dias, provocou o reaparecimento das manifestações epilépticas tal como eram observadas antes do tratamento. As tentativas de redução da medicação foram seguidas por recrudescimento das manifestações epilépticas.

Um paciente com sindrome de Lennox apresentou intensa reação à vacinação antivariólica, seguida por recidiva das crises. Tal recidiva não pôde ser controlada, pelo menos inicialmente, pelo emprêgo de maiores doses. Baseados em nossos resultados verificamos que o Ro 5-4023 apresenta campo de ação bastante amplo. Ausências típicas, mesmo rebeldes às terapêuticas com dionas e succinimidas, ausências mioclônicas e diversas manifestações epilépticas próprias da síndrome de Lennox foram beneficiadas pela droga. De interêsse é a sua ação sôbre as crises psicomotoras da infância com ponta-ondas lentas. Oller Daurella (1965) assinalou resultados "francamente favoráveis" em 30 pacientes com automatismos, não se referindo especìficamente às crises da infância cơn ponta-ondas lentas.

Pesquisas neurofisiológicas, por diversos métodos, demonstraram a ação de derivados da benzodiazepina sôbre as estruturas rinencefálicas e, particularmente, sua ação depressora sôbre o núcleo amigdalóide 1-3, 8, 22-24 indicando seu possível uso na epilepsia psicomotora. $\hat{E}$ provável que parte das manifestações clínicas observadas na síndrome de Lennox, particularmente as ausências atipicas, dependam da participação do sistema límbico.

Os exames eletrencefalográficos evidenciam, freqüentemente, predomínio das descargas de pontas-ondas lentas nas projeções temporais. O não desapa. recimento das descargas focais, particularmente as de projeção temporal e, 
eventualmente, seu aparecimento em paciente com epilepsia generalizada, pode ser devido ao fato de a ação depressora das benzodiazepinas estar essencialmente ligada ao núcleo amigdalóide, desde que foi demonstrado não ser o hipocampo deprimido por essas drogas ${ }^{8}$. Dessa forma, atividades patológicas continuam sendo registradas nas projeções temporais; porém, derivados da benzodiazepina, agindo em determinado nivel do circuito límbico, podem modificar a dinâmica dos "feed-back" dos circuitos envolvidos na produção das crises e, possìvelmente, na produção do sincronismo bilateral secundário.

De grande interêsse é o resultado obtido numa paciente com síndrome caracterizado por mioclonias de ação, mioclonias esporádicas espontâneas e convulsões tônico-clônicas generalizadas (caso 14). O tratamento com $6 \mathrm{mg}$ de Ro 5-4023 permitiu o contrôle das mioclonias intencionais durante três semanas, evidenciando-se, então, síndrome cerebelar. Tal resultado não havia sido provocado por administração de $40 \mathrm{mg} /$ dia de Diazepam e, ulteriormente, de Nitrazepam. Contudo, a paciente permaneceu cêrca de 24 horas sem manifestações após a administração endovenosa dêstes mesmos produtos. Poucas referências há na literatura a respeito do tratamento de casos semelhantes com derivados benzodiazepínicos. Declerck (1968) refere bons resultados com o uso de $10 \mathrm{mg} /$ dia de Diazepam em paciente com epilepsia de Unverricht-Lundborg.

Como tem sido observado nos seguimentos terapêticos com Diazepam e Nitrazepam a incidência de recidivas parciais com o uso de Ro 5-4023 tem sido relativamente freqüente. Assim, 8 pacientes voltaram a apresentar crises após uma a quatro semanas de tratamento, embora menos freqüentes e menos intensas.

Efeitos colaterais foram observados em metade dos pacientes. Embora, por vêzes, relativamente intensos, não justificaram a suspensão ou a redução da droga por serem passageiros em sua maioria, desaparecendo entre três a dez dias.

Seguimentos paralelos mostraram que a precipitação de crises generalizadas tônico-clônicas é maior quando se usa o Diazepam e o Nitrazepam, do que quando se emprega o Ro 5-4023.

\section{RESUMO E CONCLUSOES}

Estudaram-se as modificações do quadro clínico e do quadro eletrencefalográfico em 22 pacientes com manifestações epilépticas diárias $(5$ com ausências típicas, 3 com crises psicomotoras, 5 com ausências mioclônicas, 1 com mioclonias de ação e 8 com sindrome de Lennox), tratados com 7-nitro-5-(2clorofenil)-3H-1,4 benzodiazepina 2 (1H)-one ou Ro 5-4023. Vinte pacientes tinham sido submetidos a terapêuticas anticonvulsivantes usuais sem resultados apreciáveis. 
Com o uso de Ro 5-4023 as crises foram controladas desde o primeiro dia em 14 pacientes. Em mais 6 doentes houve redução rápida e significativa da intensidade e da freqüência das crises. Dêstes, um não mais apresentou manifestações a partir da segunda semana de tratamento. Recidivas parciais ocorreram em 8 pacientes.

Destaca-se o amplo campo de ação da droga, que age nas epilepsias generalizadas (ausências típicas e ausências mioclônicas), em epilepsias parciais, particularmente crises psicomotoras da infância com ponta-ondas lentas, nas encefalopatias epilépticas graves da infância, de tipo Lennox, e também em síndrome epiléptico degenerativo.

Os efeitos colaterais, presentes em 11 pacientes, foram passageiros em 9. Precipitação de crises generalizadas tônico-clônicas ocorreu em dois pacientes; contudo há indícios que êsse fenômeno é mais freqüente com outros derivados benzodiazepínicos.

As modificações do EEG incluiram desaparecimento ou diminuição das descargas bilaterais e difusas, espontâneas e/ou desencadeadas pela estimulação luminosa intermitente, aparecimento de rítmos rápidos e difusos, persistência ou aparecimento de anormalidades focais lentas ou de tipo irritativo.

\section{S U M M A R Y}

Longitudinal clinical and electroencephalographical studies in epileptic patients treated with Ro 5-40ミ3.

Twenty two epileptic patients, with daily seizures ( 5 had typical absences, 3 had psychomotor seizures, 5 had myoclonic absences, one had intentional myoclonia and 8 had Lennox syndrome) were treated with 7-nitro-5-(2chlorophenyl)-3H-1,4 benzodiazepine-2(1H)-one or Ro 5-4023. Twenty of these patients had received previous treatment with other anticonvulsivant drugs with no satisfactory results.

A clinical and electroencephalographical longitudinal study was made on these patients.

With the use of Ro 5-4023 the seizures were completely controlled since the first day in 14 patients; in 6 there was a rapid and significant decrease in frequency and intensity of seizures; in one, out of these 6 , seizures disappeared during the second week of treatment. Recurrence of seizures were observed in 8 patients.

The drug is effective in a large spectrum of generalized seizures (typical absences, myoclonic absences); partial seizures, in particular the psychomotor seizures of infancy with slow spike-waves; the severe epileptic encephalopathy of infancy, Lennox type; and the degenerative epileptic syndrome.

Side effects due to the drug were present in 11 patients, being of short duration in 9 . In two patients there was precipitation of generalized 
tonic-clonic seizures; anyhow it seems that the occurrence of this complication is more frequent when one uses other types of benzodiazepines.

Electroencephadographic studies showed improvement of the previous abnormalities; there was disappearance or decrease of bilateral diffuse discharges, when spontaneous or provoked by intermitent light stimuli, onset of diffuse, fast rhythm, persistance or onset of slow focal abnormalities or irritative type of discharges.

\section{REFERENCIA S}

1. ARRIGO, A.; JANN, G. \& TONALI, P. - Some aspects of the action of Valium and of Librium on the electrical activity of the rabbit brain. Arch. Intern. Pharmacodyn. 154:364, 1965.

2. BRAZIER, M. A. B. - The effects of drugs on the electroencephalogram in man. Clin. Pharmacol. Ther. 5:102, 1964.

3. DECLERCK, A. - Diagnostique et traitement de l'épilepsie myoclonique progressive Unverricht-Lundborg. Acta Neurol. Belg. 68:471, 1969.

4. EIDELBERG, E.; NEER, H. M. \& MILlER, M. K. - Anticonvulsant properties of some benzodiazepine derivatives; possible use against psychomotor seizures. Neurology (Minn.) 15:223, 1965.

5. Gastaut, H.; ROGER, J.; TIMSIT, M. \& BROUGHTON, R. - An electroclinical study of generalized epileptic seizures of tonic expression. Epilepsia (Amst.) 4:15, 1963.

6. GASTAUT, H.; ROGER, J.; SOULAYROL, R.; LOB, H. \& TASSINARI, C. A. - L'action du diazépan (Valium) dans le traitement des formes non convulsives de l'épilepsia généralisée. Rev. Neurol. (Paris) 112:99, 1965.

7. GIBBS, F. A. \& ANDERSON, E. M. - Treatment of hypsarhythmia and infantile spasms with a Librium analogue. Neurology (Minn.) 15:1173, 1965.

8. HIMWICH, H. E.; MORILlO, A. \& STEINER, W. G. - Drugs affecting rhinencephalic structures. J. Neuropsychiat. (Chic.) supl. 3:15, 1962.

9. JONG, T. H. - Klinishe Erfahrungen mit dem Benzodiazepin derivat Ro 4-5360 bei der Behandlung der Epilepsie. Schweiz. med. Wschr. 94:1, 1964.

10. LEFEVRE, A. B. \& GAZAL, S. - Tratamento do estado de mal epiléptico em crianças pelo Valium. Arq. Ncuro-Psiquiat. (São Paulo) 25:155, 1968.

11. LEFévre, A. B. - Tratamento da epilepsia na infãncia com Mogadon. Arq. Neuro-Psiquiat. (São Pauk) 27:109, 1969.

12. LISKE, E. \& FORSTER, F. M. - Clinical study of a new benzodiazepine as an anticonvulsant agent. J. New' Drugs (New York) 3:241, 1963.

13. LISON, M. P. \& MECA, D. - Espasmos em flexão: estudo clínico e eletrencefalográfico em pacientes tratados com Ro 4-5360. Arq. Neuro-Psiquiat. (São Paulo) 25:87, 1967.

14. LISON, M. P. \& FASSONI, L. F. - Emprêgo de Mogadon endovenoso em pacientes epilépticos. Estudo clínico e eletrencefalográfico. Arq. Neuro-Psiquiat. (São Paulo) 26:341, 1968. 
15. MARKHAM, C. H. - The treatment of myocionic seizures of infancy and childhood with LA-1. Pediatrics 34:511, 1964.

16. MEGA, D. \& LISON, M. P. - Valium endovenoso no tratamento do estado de mal epiléptico. Arq. Neuro Psiquiat. (São Paulo) 26:1445, 1968.

17. MILliCHAP, J. G. \& ORTIZ, W. R. - Nitrazepam in myoclonic epilepsies. Am. J. Dis. Child. 112:242, 1966.

18. MIRIBEL, J. \& MARINIER, R. - Résultats thérapeutiques de quelques benzodiazépines en prise chronique dans l'épilepsie grave de l'enfant. J. Med. Lyon 47:1584, 1966.

19. Nieto, D.; CASTRO, M.; CARbajAL, L.; ESTRADA, J.; HURTADo, A.; PEREZ JIMENEZ, E. \& MOLINA, F. - Tratamiento de la epilepsia con nuevo derivado de la benzodiazepina (Valium Ro 5-2807). Neurol. Neurocir. Psiquiatr. (México) 3:168, 1962.

20. OlLER-DAURELla, L. - El Valium y el Mogadon en el tratamiento de los automatismos. Rev. Esp. Otoneuro-oftalm e Neurocir. 24:233, 1965.

21. PETERSON, W. G. - Clinical study of Mogadon; a new anticonvulsant. Neurology (Minn.) 17:878, 1967.

22. RANDALL, L. O.; SCHALLEK, W.; SCHECKEL, C.; BANZIGER, R.; BORIS, A.; MOE, R. A.; BAGDON, R. E.; SCHWARTZ, M. A. \& ZBINDEN, G. - Zur Pharmakologie von Valium einem neuen Psychopharmakon der Benzodiazepinreike. Schweiz. Med. Wschr. 93:794, 1963.

23. SCHALLEK, W.; KUEHN, A. \& JEW, N. - Effects of chlordiazepoxide (Librium) and other psychotropic agents on the limbic system of the brain. Ann. New York Acad. Med. 96:303, 1962.

24. SCHALLEK, W. \& KUEHN, A. - Effects of trimethadione, diphenylhydantoin and chlordiazepoxide on after-discharger in brain of cat. Proc. Soc. Exp. Biol. (New York) 112:813, 1963.

25. SNYDER, C. H. - Myoclonic epilepsy in cildren: short term comparative study of two benzodiazepine derivatives in treatment. South. Med. J. (Birmingham) 61:17, 1968.

26. SWINYARD, E. A. \& CASTELlion, A. W. - Anticonvulsant properties of some benzodiazepines. J. Pharmac. Exp. Therap. 151:369, 1966.

27. TROLLE, E. - Diazepam (Valium) in the treatment of epilepsy; a report of fifty cases. Acta. Neurol. Scandinav. 41:535, 1965.

28. VOLZKE, E.; DOOSE, H. \& STEPHAN, E. - The treatment of infantile spasms and hypsarhythmia with Mogadon. Epilepsia (Amst.) 8:64, 1967.

29. 'WEINBERG, W. A. \& HARWELL, J. L. - Diazepam (Valium) in myoclonic seizures: favorable response during infancy and cildhood. Am. J. Dis. Child. 109:123, 1965.

30. WEINMAN, H. M. - Le traitement des crises infantiles par le Mogadon. Rev. Neurol. (Paris) 117:152, 1967.

Departamento de Neurologia - Faculdade de Medicina de Ribeirão Prêto - Ribeirão Prêto, SP - Brasil. 